\title{
Zur Theorie des Mechanismus des künstlichen Gelenks.
}

\author{
Von \\ Dipl.-Ing. W. v. Schiitz, \\ Botriebsleiter an der Prüistello für Ersatzolieder, Berlin-Charlottenburg.
}

Nit 3 tbbildungen im Text.

(Eingegangen am 21. September 1919.)

In seinem Aufsatz our "Theorie des Mechanismus des künstlichen Gelenks" in Band 17, Heft 1 dieses Archivs will Wildermuth einen neuen Weg angeben, wie die Frage der Normalisierung der künstlichen Glieder zu lösen ist Es isu dazu folgendes zu bemerken:

Das Bestreben, im Ersatzgliederbau zu normalisieren, ist so alt und älter (Hoeftmánn, Rohr-Hilden) wie die Prüfstellen. Die Charlottenburgor Prüfstelle ist am 1. Februar 1916 georündet, und im Mai 1916 ist bereits das Merkblatt Nr. 2 mit den Nornalien der Schrambengewinde und der Befestigungszapfen der Ansatzstücke herausgegeben worden Die Darstellung Wildermutha, es sei die Notwendigkeit der Normalisierung erst jetzt erkannt von den verschiedenen Prüfstellen wird immer mehr dio Notwendigkeit betont, beim Ersatzgliederbau auf eine gewisso Vereinheitlichung der Grundelemente zu achten - ist demnach unzutreffend.

Daß sich 1. im allgemeinen Normalien ohne Zwangsmittel schwer einführen lassen, liegt an dem Widerstand einzelner Firmen, die durch Umstellung ihrer Fabrikation große Unkosten haben.

Daß 2. weitere Formalien nicht herausgegeben wurden, liegt an folgenden Gründen :

Dio Normalisierung eines Bauelementes ist gleichzeitig der Abschluß seiner Entwicklung für eine längere Zeit oder für immer. Es ist daher nicht geraten, zu normalisieren, ehe genügend Erfahrungen vorliegen und die Entwicklung bis zu einem gewissen Grade abgeschlossen ist. Die von Wildermuth erwähnte Normalisierung der Bein-Einzelteile ist sehr erwünscht; der Zeitpinkt ist unbedingt gekommen, jedoch aus anderen Gründen.

Die Amputierten stellen mit Pecht die Forderung:

1. leicht answechselbare normalisierte Teile an ihren Kunstbeinen zu haben, die sie bei jedem Bandagisten erhalten körnen,

2. die Instandhaltung ihres Kunstbeines möglichst einfach und bequem selbst vornehmen zu können, auch bei verschiedenen Beinsystemen. 
Im übrigen ist der Staat an der durch die Normalisiernug zu erreichenden Verbilligung der künstlichen Beine lebhaft interessiert.

Wenn richtig normalisiert wird, daun stelli der normalisierte Teil gleichzeitig die Summe aller praktischen Erfahrungen dar. Wie duch die Cntersuchumgen Wildermuths die Normalisierung gefördert wird, ist ohne weiteres nicht ersichtlich. Wilclermuth geht, anch später in dem bisher erschienenen Teil seines Aufsatzes über das Fillbogengelenk (der doch wohl bezüglich des letztoren als abgeschlossenes Ganze anzusehen ist) darauf nicht mehr ein.

Luabhängig von der Frage der Normalisierung scheint Wildermuth in der Hauptsache mechanische Gesetze aufstellen zu wollen, die gleichzeitig anf das mechannische wie auf das künstliche Gelenk anwendbar sind. Es wird also versucht, Muskelmechanik, die ein Teil der speziellen Muskel-Physiologie ist, nit der H[echanik künsticher Gelenke zu vermengen. Ieider führt der Verstch nicht zu dem gewinschten Ziel, da ctie mechanischen IJedingungen für natürliche und künstliche Gelenke nicht ohne weiteres miteinander vergleichbar sind.

Im einzolnen sei auf folgende Mangel hingewiesen:

Der Begriff der Kinematik wird gleich im Anfang verstümmelt, indem Wildermuth schreibt, daß er unter Kinematik nicht nur die Lehre von den Bewegungsworgingen, sondern auch die diese Bewegung bewirkenden Kräfte versteht. Aus den folgenden Ausfiuhrungen Wildermuths geht hervor, dals für "Kinematik" besser ,Wechanik" 2.1 setzen ist. Die Begriffe im einze]nen 7.u erläutern, ist hier nicht der Platz. Wis finden sich derartige sehr klare Bcgriffisestimmungen z. B. in du Bois-Reymond: Spezielle Muskel-Physiologie oder Bewegungslehre.

Reuleaux spricht auch nicht von zwangsmißigen Bewegungen, sondern son Zwanglauf. Zwanglauf ist am menschlichen Körper nach dem Begriff der Kinematik nicht vochanden. Han kömnte hier höhstens von Kraftschluß sprechen, denn saimtliche Gelenkteile werden durch die Kraft, die den Gelenkbändern und Wuskehn innewohnt, zu einem Gauzen zusammengehalten. Ein Kuochenskelett fällt anseinander, wenn man nicht church Drailte für Verbindung ler Knochen unteroinander sorot. Wan kamn z. B. vom Rastengelenk-Arm sagen, die einzelnen Teile sind zwanglanfig mitcinander verbunden; beim Reibungsgelenk \%. B. ist auch nu Kraftschluß vorhanden, da die Reibungskräfto an der Chertragung von Kraften einen wesentlichen Anteil haben.

Dio Betrachêugsworist der natülichen Gelenke narh dem Freiheitsgrad der Bewegung ist nicht nen whe bereits von du Bois-Reymond in seinem oben erwihhnten Buche (Seite 94) angewandt worden. Die Betrachtungsweise sehort auch nicht in das Gebiet dor Mathematik, wie Wildermuth angibt. sondern ist ein Teil der Geometrie der Bewegungen (siehe Scholl, Theorie der Bewegung und der Krifte, Jcip\%ig 1s70 und Situdy: Goometrie der Dynamen, Leipzig 1903). Diese Botrachtungsweise ist also durchans Gemeingut der Technik und jedem Ingenieur geläufig. Fs erscheint angebracht, darauf hinzuweisen, wie systematisch der Ingeniour vorgeht, $\mathrm{m}$ mit seinen technischen Begriffen das natürliche Gelenk zu erfassen und aus dem natürlichen Gelent die Bewegnngsbedingungen für das künstliche? Gelenk abzuleiten. (Siehe Handbuch der Prüfstelle Charlottenburg: ,Ersateglieder und Arbeitshilfon für Kriegs- 
beschädigte und Unfallverletzte", Schlesinger: "Der mechanische Aufbau der künstlichen Glieder", Seite 374). Die natürlichen Armgelenke und ihr mechanischer Ersatz werden nebeneinander gestellt, ohne den Umweg über alle schwjerigen Begriffsbestimmungen zu nehmen. Wir müssen ja doch die Gelenke aus unserem Erfahrungsgebiet nehmen. Für den praktischen Ersatzgliederbau kann es also höchstens interessant sein zu wissen, duß irgend ein Gelenk dem 1.-6. Freiheitsgrad angehör. Eine unmittelbare Förderung des Banes künstlicher Gelenke ist aber hieraus nicht zu erhoffen.

Neu ist der Begriff ,Iaufwiderstand", doch trage ich gegen seine allgemeine Einführung schwero Bedenten, da mit dem Begriff:

1. sowohl für das natürlicho wie für das künstliche Gelenk keine klare mechanische Vorstellung verbunden ist,

2. für das künstliche Gelenk der Begriff "Reibungswiderstand" besteht und ein Ersatz hierfür wenigstens zunächst nicht notwendig erscheint.

Den Kraftwirliungen bei den von Wildermuth gewällten Beispielen des nutürlichen Ellbogengelenkes und des Reibungsgelenkes als Ellbogengelerk liegen ganz verschiedene mechanische Vorstellungen zugrunde. Der Unterschied geht aus den folgenden Beispielen hervor:

\section{A. Das natïrliche Gelenk.}

Sehen wir bei dem natülichen Gelenk von der Pro- und Supinationsbewegung ab, damn wirkt eine Muskelgruppe als Beuger, die zweite als Strecker.

In Abb. I seien Ober- und Lnterarm durch starre, zunächst gewichtslose Stïbe $O$ und $\mathrm{C}$ dargestellt. $O$ sei bei $\mathrm{K}$ fest eingespannt gedacht.

Es sei :

$P_{b}$ die Kraft, die die Beugergruppe auf den Unterarm ausübt,

$b$ der Hebelarm der Kraft $P_{b}$, ausübt,

$P_{a}$ die Kraft, die die Streckergruppe

c der Hebelarm der Kraft $P_{9}$,

$Q$ die am distalen Ende des Unterarmes etwa in der Handfläche wirkende liast,

a der Hebelarm der Last $Q$.

Fis besteht dic Momentengleichung: $\mathrm{Q} \cdot \mathrm{a}+\mathrm{P}_{\mathrm{g}} \cdot \mathrm{c}=\mathrm{P}_{\mathrm{b}} \cdot \mathrm{b}$, wenn das System im Gleichgewicht sein

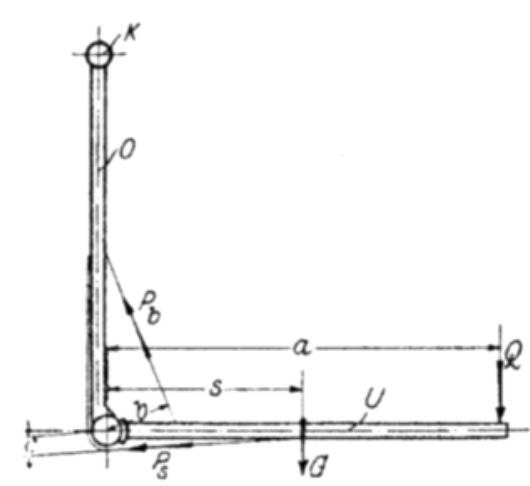

Abb. 1. soll. Bei Berücksichtigung des Unterarmgewichtes $G$, wirkend im Sichwerpunktsabstand s, wärde die Gleichung folgende Form annehmen:

$$
\mathrm{Q} \cdot \mathrm{a}+\mathrm{P}_{\mathrm{a}} \cdot \mathrm{c}+\mathrm{G} \cdot \mathrm{s}=\mathrm{P}_{\mathrm{b}} \cdot \mathrm{b} .
$$

Die Kraft der Beugergruppe, wirkend am Hebelarm b, ergibt ein Drehmoment, das deu übrigen auftretenden Drehmomenten entgegenwirkt; von der Gelenkreibung wird hier, wie es auch Wildermuth tut, absichtlich abgesehen. 


\section{B. Das kïnstliche Gelenk.}

Beim künstlichen Gelenk (im rorigen Falle Reibungsgelenk) ergibt die mechanische Vorstelhung cin ganz anderes Bild. Da es sich nur um prinzipielle Richtigstellung hardelt, sei es gestattet, die Anschaumgsweise dor Mechanik nur an einem Beispiel zu besprechen.

Lch benutze die Gelenkkonstruktion Wildermuths (siehe Seite 86 dieses Archivs, Band 17, Heft 1, Abb. 1-3) und betrachte auch hier wie in dem vorigen Beispiel nur die Bengebewegung.

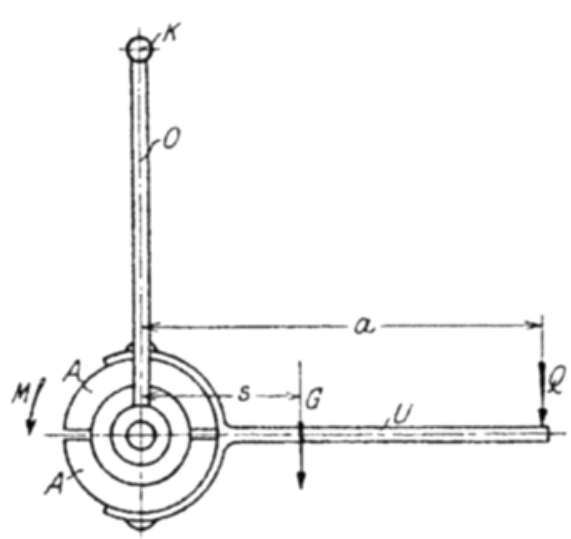

Abb. 2.

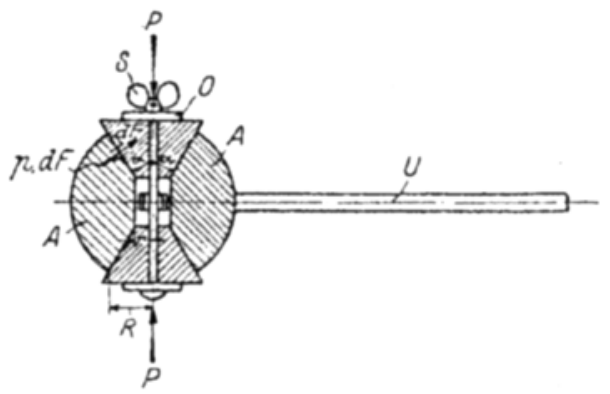

Abb. 3 .

Für diesen Fall läßt sich die Konstruktion Wildermuths durch folgendes Schema (Abb. 2 und 3) ersetzen.

Zwischen den Oberarmschienen $O$ befinden sich auf der Ellbogenachsc zwei kegelstumpfförmige Körper, die durch Sichraube $S$ in konische Ausdrehungen dor Kugelteile A gepreBt werden. Kugelteile A seien mit den Unterarmschienen $U$ für diese Betrachtung fest verbunden ${ }^{1}$ ). Die an den Kegelstumpfoberflächen beim Anziohen der Schraube S entstehende Reibung erzeugt ein Drehmoment, das dem durch die Last $Q$ erzeugten Drehmoment entgegenwirkt.

Es sei:

M das durch die Reibung erreugte Drehmoment,

$Q$ wiederum dio in der Hand-

fliche wirkendo Tast,

a der Hobelarm der last $Q$.

Datn ist nach dem obigen

$$
\mathrm{M}=\mathrm{Q} \text {. a. }
$$

Dieses Moment M liab sich anf folgende Weise bestimmen:

Zunïchst wird auf jeden der beiden Kégelstümpfe in Richinng der Schraubenachse die durch den Anzug der Sichmube erzengte Kraft P ausgeübt. Das gewamte Drehmoment der Reibung M zerfält dementsprechend in zwei gleiche Reibungsmomente $\mathrm{M}^{\prime}$, also $\mathrm{M}=2 \mathrm{Wl}^{\prime}$.

Es sei:

d $F$ cin unendlich kleines Flïhenteilchen einer Kegelstumpfoberfliche, $p$ dor Druck auf die Flicheneinheit.

Damn ist $\mathrm{p} . \mathrm{d} \mathrm{F}$ die auf ein Flühenteilchen d $\mathrm{F}$ ausgeübte Druckkraft.

1) Bei der Konstraktion W.'s sind die Unterarmschie: en auf dern Kugelteil A drehbar, un die Sichelluewogung mormöglichen. Fïr obige prinzipinllo Betrachtung wurde von der Sichelbewegung ibgesehen. 
Furner sei:

f der Reibungsfaktor,

$\varrho$ der jeweilige Hebelarm von $\mathrm{p} . \mathrm{d} \mathrm{F}$; dann ist

$$
\mathrm{H}^{\prime}=\int \mathrm{f} \cdot \mathrm{Q} \mathrm{P} \cdot \mathrm{d} \mathrm{F} \text {. }
$$

Forner sei:

$R$ der Radius des gröBeren Berührungskreises des Kegelstumpfs,

$r$ der Radius des kleinen Berührungskreises des Kegelstumpts,

a dor Kcgelwinkel des Kegelstumpfs, so wird nach Keck: Mechanik I, Hannover 1900:

$$
\mathrm{H}^{\prime}=-\frac{2}{3} \mathrm{P} \cdot \frac{\mathrm{f}}{\sin a} \cdot \frac{\mathrm{R}^{3}-\mathrm{r}^{3}}{\mathrm{R}^{2}-\mathrm{r}^{2}}
$$

und

$$
M=Q \cdot a=\frac{4}{3} \cdot P \cdot \frac{f}{\sin \alpha} \cdot \frac{P^{3}-r^{3}}{R^{2}-r^{2}} .
$$

Bei Berüchsichtigung des Enterarmgewichts

$$
Q \cdot a=\frac{4}{3} \cdot P \cdot \frac{f}{\sin a} \cdot \frac{R^{3}-r^{3}}{R^{2}-r^{2}}-G \cdot s .
$$

Wir finden also für das natürliche Celenk:

$$
\mathrm{Q} \cdot \mathrm{a}=\mathrm{P}_{\mathrm{b}} \cdot \mathrm{b}-\mathrm{P}_{\mathrm{s}} \cdot \mathrm{c}-\mathrm{G} \cdot \mathrm{s},
$$

für das küustliche Gelenk:

$$
\mathrm{Q} \cdot \mathrm{a}=\frac{4}{3} \mathrm{P} \cdot \frac{\mathrm{f}}{\sin u} \cdot \frac{\mathrm{R}^{3}-\mathrm{r}^{3}}{\mathrm{R}^{2}-\mathrm{r}^{2}}-\mathrm{G} \cdot \mathrm{s} .
$$

Wildermuth schreibt: „Unter Laufwiderstand verstehe ich die Kraft, die einer Bewegung der Gelenkteile entgegenwirkt usw. . . Da es sich sowohl beim tierischen wie bcim mechanischen Gelenk un gleitende Reibung handelt, so läßt sich der Laufwiderstand durch die Formel:

$$
\mathrm{L}=\mathrm{f} . \mathrm{P}
$$

wiedergeben, wobei $\mathrm{L}$ den in irgend einem Punkte des Gelenkes angreifonden Gesamt-Widerstand, f der Reibungszahl und P den auf dem Gelenk lastenden Druck entspricht. Das tierische Gelenk ist praktisch reibungsfrei."

Von der Reibung in tierischen Gelenk wurde bei der Entwjcklung der Formel 1 ausdrücklich abgesehen. Der Vergleich der Formeln 1 und 2 zeigt aber schon den grundsätzlichen Untersehied der mechanischen Verhältnisso beim künstlichen und natürlichen Gelenk, so daß für beide eine gerneinsame Definition durch die Formel:

$$
\mathrm{L}=\mathrm{f} . \mathrm{P}
$$

vine Unmöglichkeit ist. Den Faktor $f$ bezeichnet Wildermuth einmal richtig als den Reibungsfaktor, nämlich eine Zahl, die je nach Art der Oberfläche, Material, Raubigkeit, Schmieruug verschieden ist. Später wird $f$ als die Reibung gemeinhin bezeichnet, nämlich - ,Die Reibung (S. 81, Band 17, Heft 1 dieses Archivs), d. h. der Faktor $f$ in der Formel $L==f . P$ tritt hier (beim 
natürlichen Gelenk) also ganz zurück,"-- eine Bezcichnung, die nur aus der ohnehin oberflächlichen Formulieruug $I_{2}=\mathrm{f}$. P z.1ı erklären ist.

Welche Vorstellung verbindet sich mit der Frklärung von $\mathrm{L}$ als dem in irgend einem Punkte des Gelenkes angrejfenden Gesamtwiderstand?

Wahrscheinlich gar keine, denn beim natürlichen Gelenk sind es Muskelkräfte, die an Hebelarmen wirken, beim künstlichen Gelenk sind es die Reibungsmomente, die auf den Kegelstumpfoberflächen entstehen, wenn die Schraube $\mathrm{S}$ angezogen wird.

P ist bei Wildermuth der auf dem Gelenk lastende Druck. Für das natürliche Gelenk ist eine Anwendung von $P$, da die Reibung unberücksichtigt bleiben soll, nicht möglich; für das künstliche Gelenk haben wir weiter oben den Begriff p.dF einführen mïssen als den Druek pro Flächenteilchen d F.

Eine gerneinsame Erklärung der mechanischen Vorgärge beim künstFichen und natürlichen Gelenk durch $L=f . P$ ist somit ebensowenig möglich wie die gemeinsame Bezeichmung "Ianfwiderstand". Die Grüude hierfür sind oben schon vorweg genommen und durch die Beispiele erwiesen. 